PROCEEDINGS OF THE AMERICAN MATHEMATICAL SOCIETY

Volume 124, Number 8, August 1996

\title{
A SUBMARTINGALE INEQUALITY
}

\author{
CHANGSUN CHOI
}

(Communicated by Richard Durrett)

\begin{abstract}
This paper extends Burkholder's inequality between a nonnegative submartingale and a process strongly differentially subordinate to it.
\end{abstract}

\section{Statement of the ineQuality}

Let $0 \leq \alpha \leq 1$ and $1<p<\infty$. Suppose that $f=\left(f_{n}\right)_{n \geq 0}$ and $g=\left(g_{n}\right)_{n \geq 0}$ are adapted to a filtration $\left(\mathcal{F}_{n}\right)_{n \geq 0}$ of a probability space $(\Omega, \mathcal{F}, P)$. Here $f$ is a nonnegative submartingale and $g$ is $\mathbb{R}^{\nu}$-valued, where $\nu$ is a positive integer. With $f_{n}=d_{0}+\cdots+d_{n}$ and $g_{n}=e_{0}+\cdots+e_{n}(n \geq 0)$ we assume that

$$
\begin{aligned}
\left|e_{n}\right| & \leq\left|d_{n}\right| \quad(n \geq 0), \\
\left|\mathbb{E}\left(e_{n} \mid \mathcal{F}_{n-1}\right)\right| & \leq \alpha \mathbb{E}\left(d_{n} \mid \mathcal{F}_{n-1}\right) \quad(n \geq 1) .
\end{aligned}
$$

Then, with $\|f\|_{p}=\sup _{n \geq 0}\left\|f_{n}\right\|_{p}$, we have the inequality

$$
\|g\|_{p} \leq(r-1)\|f\|_{p}
$$

where $r=\max \{(\alpha+1) p, p /(p-1)\}$ and $r$ is best possible.

Remarks. 1. The case $\alpha=1$ is shown in Burkholder's paper [3].

2. For martingales $f$ and $g$ that satisfy (1.1) and have values in a Hilbert space, the inequality (1.3) is shown in Burkholder's paper [2]. There $r=\max \{p, p /(p-1)\}$.

3. Best possible means that if $r-1$ in (1.3) is replaced by a strictly smaller number $\beta$, then the opposite inequality $\|g\|_{p}>\beta\|f\|_{p}$ holds for some probability space and some $f$ and $g$ as above.

\section{Outline of the PRoOF OF THE INEQUALITY}

We may assume $\|f\|_{p}<\infty$. Also, we may assume

$$
f_{n-1}>0 \text { and }\left|g_{n-1}+t e_{n}\right|>0 \text { for all } t \in \mathbb{R} \text { and } n \geq 1 .
$$

Indeed, for each $0<\varepsilon<1$, the processes $f^{\varepsilon}$ and $g^{\varepsilon}$, where $f_{n}^{\varepsilon}=f_{n}+\varepsilon$ and $g^{\varepsilon}=\left(g_{n}, \varepsilon\right)$, satisfy $(2.1)$ and all the assumptions in Section 1 . Here $g^{\varepsilon}$ is a process in $\mathbb{R}^{\nu+1}$. Since $\|g\|_{p} \leq\left\|g^{\varepsilon}\right\|_{p}$ and $\left\|f^{\varepsilon}\right\|_{p} \leq\|f\|_{p}+\varepsilon$, the inequality (1.3) follows from $\left\|g^{\varepsilon}\right\|_{p} \leq(r-1)\left\|f^{\varepsilon}\right\|_{p}$ as $\varepsilon$ tends to 0 .

Received by the editors February 1, 1995.

1991 Mathematics Subject Classification. Primary 60G42; Secondary 60E15.

Key words and phrases. Submartingale, differential subordination, conditional differential subordination, norm inequality.

(C)1996 American Mathematical Society 
Put $S=(0, \infty) \times \mathbb{R}^{\nu}$ and define functions $U$ and $V$ on $S$ by

$$
\begin{gathered}
U(x, y)=(|y|-(r-1) x)(x+|y|)^{p-1}, \\
V(x, y)=|y|^{p}-((r-1) x)^{p} .
\end{gathered}
$$

Then the inequality (1.3) follows from

$$
\mathbb{E} V\left(f_{n}, g_{n}\right) \leq 0 \text { for all } n \geq 0
$$

which is a consequence of the inequalities:

$$
\begin{gathered}
\mathbb{E} V\left(f_{n}, g_{n}\right) \leq p(1-1 / r)^{p-1} \mathbb{E} U\left(f_{n}, g_{n}\right) \quad \text { for all } n \geq 0, \\
\mathbb{E} U\left(f_{n}, g_{n}\right) \leq \mathbb{E} U\left(f_{n-1}, g_{n-1}\right) \quad \text { for all } n \geq 1, \\
\mathbb{E} U\left(f_{0}, g_{0}\right) \leq 0 .
\end{gathered}
$$

\section{Proof of the inequality}

It remains to prove the inequalities (2.5), (2.6) and (2.7).

Proof of (2.5). Notice that (2.5) is proved if $V(x, y) \leq p(1-1 / r)^{p-1} U(x, y)$ for all $(x, y) \in S$, and that the latter inequality holds if and only if it holds with $x$ replaced by $x /(x+|y|)$ and $y$ replaced by $y /(x+|y|)$. Thus, it is enough to prove the inequality for $x \in(0,1]$ and $|y|=1-x$, or, equivalently, to prove $F \leq 0$ on $[0,1]$ where

$$
F(x)=(1-x)^{p}-[(r-1) x]^{p}-p(1-r x)(1-1 / r)^{p-1} .
$$

If $0<x<1$, then

$$
\begin{gathered}
F^{\prime}(x)=-p\left[(1-x)^{p-1}+(r-1)^{p} x^{p-1}\right]+r p(1-1 / r)^{p-1}, \\
F^{\prime \prime}(x)=p(p-1)\left[(1-x)^{p-2}-(r-1)^{p} x^{p-2}\right] .
\end{gathered}
$$

Notice that $0<1 / r<1$ and $F(1 / r)=F^{\prime}(1 / r)=0$.

If $p=2$, then $F^{\prime \prime}(x)=2\left[1-(r-1)^{2}\right] \leq 0$ on $(0,1)$ because $r \geq(\alpha+1) 2 \geq 2$. Thus $F$ has the maximum at $x=1 / r$ which proves $F \leq 0$ on $[0,1]$ in this case.

Now assume $1<p<2$. The strict concavity of $\log x$ implies that

$$
\log 1>a \log a+(1-a) \log (a+1) \quad \text { or } \quad(a+1)^{a-1}>a^{a} \quad \text { for } 0<a<1,
$$

so $a=p-1$ gives $(p-1)^{p-1}<p^{p-2}$. Hence $r^{p-1} \geq(p /(p-1))^{p-1}>p$. Thus $F(1)=-(r-1)^{p}-p(1-r)(1-1 / r)^{p-1}=\left(p-r^{p-1}\right)(r-1)^{p} / r^{p-1}<0$. Let $x^{*}$ be the zero of $F^{\prime \prime}$. Computation gives $x^{*}\left[1+(r-1)^{p /(p-2)}\right]=1,0<1 / r<x^{*}<1$, and $F^{\prime \prime}(x)<0$ if $0<x<x^{*}$. Thus the maximum of $F$ on $\left[0, x^{*}\right]$ is $F(1 / r)=0$. Also, $F^{\prime \prime}(x)>0$ if $x^{*}<x<1$. Thus $F$ is convex on $\left[x^{*}, 1\right]$, hence $F \leq 0$ on $\left[x^{*}, 1\right]$; because $F\left(x^{*}\right) \leq 0$ and $F(1)<0$.

The case $p>2$ is handled similarly. Here $F(0)<0$ follows from the inequality

$$
(a+1)^{a-1}<a^{a} \text { for } a>1
$$

which follows from (3.4) upon replacing $a$ by $1 / a$.

Proof of (2.6). The proof depends on the inequality: If $(x, y)$ and $(x+h, y+k)$ belong to $(0, \infty) \times \mathbb{R}^{\nu}$ with $|h| \geq|k|$, and $y+t k \neq 0$ for all $t \in \mathbb{R}$, then

$$
U(x+h, y+k) \leq U(x, y)+\varphi(x, y) h+\psi(x, y) \cdot k
$$


where $\varphi(x, y)=U_{x}(x, y)$ and $\psi(x, y)=U_{y}(x, y)$, that is,

$$
\begin{aligned}
& \varphi(x, y)=[(p-r)(x+|y|)-(p-1) r x](x+|y|)^{p-2}, \\
& \psi(x, y)=[p(x+|y|)-(p-1) r x](x+|y|)^{p-2} y /|y| .
\end{aligned}
$$

For a proof of (3.6) in the case $\alpha=1$, see [3]; the case $0 \leq \alpha<1$ requires only the further observation that $r \geq p /(p-1)$ and $r \geq p$.

Now (2.1) and (3.6) give

$$
U\left(f_{n}, g_{n}\right) \leq U\left(f_{n-1}, g_{n-1}\right)+\varphi\left(f_{n-1}, g_{n-1}\right) d_{n}+\psi\left(f_{n-1}, g_{n-1}\right) \cdot e_{n}
$$

where all the random variables are integrable: for example the last one is integrable because of the assumption (1.1) and the estimate $|\psi(x, y)| \leq \operatorname{pr}(x+|y|)^{p-1}$. Conditioning on $\mathcal{F}_{n-1}$ and using the assumption (1.2), one sees from (3.7) that

$$
\begin{aligned}
& \mathbb{E} U\left(f_{n}, g_{n}\right)-\mathbb{E} U\left(f_{n-1}, g_{n-1}\right) \\
& \quad \leq \mathbb{E}\left(\left[\varphi\left(f_{n-1}, g_{n-1}\right)+\alpha\left|\psi\left(f_{n-1}, g_{n-1}\right)\right|\right] \mathbb{E}\left(d_{n} \mid \mathcal{F}_{n-1}\right)\right) .
\end{aligned}
$$

Here $f$ is a submartingale, hence $\mathbb{E}\left(d_{n} \mid \mathcal{F}_{n-1}\right) \geq 0$. Thus the inequality (2.6) follows from the inequality $\varphi(x, y)+\alpha|\psi(x, y)| \leq 0$ which, by homogeneity, is equivalent to the inequality $G(x) \leq 0$ if $0 \leq x \leq 1$, where

$$
G(x)=(p-r)-(p-1) r x+\alpha|p-(p-1) r x| .
$$

Clearly $G$ is convex, hence it suffices to show $G(0) \leq 0$ and $G(1) \leq 0$. Here $G(0)=(\alpha+1) p-r \leq 0$, and noting that $p-(p-1) r \leq 0$, one has

$$
\begin{aligned}
G(1) & =(p-r)-(p-1) r-\alpha[p-(p-1) r] \\
& =-r+(1-\alpha)[p-(p-1) r] \leq 0 .
\end{aligned}
$$

This proves (2.6).

Proof of (2.7). Since $r \geq 2$ we have $|y|-(r-1) x \leq|y|-x \leq 0$ if $|y| \leq x$. Hence (2.7) follows from (2.2), the assumption (1.1) which gives $\left|g_{0}\right| \leq\left|f_{0}\right|$ when $n=0$, and the nonnegativity of $f_{0}$.

\section{Sharpness of the INEQUALITY}

Case 1. Suppose that $1<p \leq(\alpha+2) /(\alpha+1)$. Then $r=p /(p-1)$ and so $r-1$ is the best constant in (1.3) since it is already the best possible constant if $f$ is a nonnegative martingale as can be seen in (5.90) and (5.91) of [1].

Case 2. Here $p>(\alpha+2) /(\alpha+1)$ so $r=(\alpha+1) p$. Choose a small $\eta>0$ so that $\eta(p-1)(\alpha+1)<2$. Define $\left(x_{n}\right)_{n \geq 1}$ and $\left(\pi_{n}\right)_{n \geq 1}$ by

$$
r x_{n}=2+n(\alpha+1) \eta \quad \text { and } \quad \pi_{1}=\frac{1}{2}, \pi_{n+1}=\frac{1}{2} \prod_{k=1}^{n}\left(1-\frac{\eta}{x_{k}}\right) .
$$

Now we define a filtration $\left(\mathcal{F}_{n}\right)_{n \geq 0}$ on the Lebesgue probability space $[0,1)$ as follows: $\mathcal{F}_{0}=\{\varnothing,[0,1)\}$ and for $n \geq 1, \mathcal{F}_{2 n-1}=\mathcal{F}_{2 n}$ is generated by the partition of $[0,1)$ determined by $0<\pi_{n}<\pi_{n-1}<\cdots<\pi_{1}<1$. 
Using the same notation for the interval $[a, b)$ and its characteristic function on $[0,1)$ we put

$$
\begin{aligned}
& d_{0}=e_{0}=[0,1), \quad d_{1}=-e_{1}=-\left[0, \frac{1}{2}\right)+\left[\frac{1}{2}, 1\right), \\
& d_{2 n}=\eta\left[0, \pi_{n}\right), \quad e_{2 n}=\alpha d_{2 n}, \\
& d_{2 n+1}=-e_{2 n+1}=-\eta\left[0, \pi_{n+1}\right)+\left(x_{n}-\eta\right)\left[\pi_{n+1}, \pi_{n}\right) .
\end{aligned}
$$

Let $f_{n}=d_{0}+\cdots+d_{n}$ and $g_{n}=e_{0}+\cdots+e_{n}$. Then one checks that $f$ and $g$ are adapted to $\left(\mathcal{F}_{n}\right)$, that $f$ is a nonnegative submartingale, and that (1.1) and (1.2) are satisfied. Also,

$$
\begin{aligned}
& f_{2 n+1}=\sum_{1 \leq k \leq n} x_{k}\left[\pi_{k+1}, \pi_{k}\right)+2\left[\frac{1}{2}, 1\right), \\
& g_{2 n+1}=r x_{n}\left[0, \pi_{n+1}\right)+(r-1) \sum_{k=1}^{n} x_{k}\left[\pi_{k+1}, \pi_{k}\right) .
\end{aligned}
$$

Thus we get

$$
\left\|g_{2 n+1}\right\|_{p} \geq(r-1)\left\|f_{2 n+1}\right\|_{p}\left(\frac{A_{n}}{A_{n}+2^{p}}\right)^{1 / p}
$$

with $A_{n}=a_{1}+\cdots+a_{n}$ where $a_{n}=\left|x_{n}\right|^{p}\left(\pi_{n}-\pi_{n+1}\right)$.

Notice that each pair of stopped processes, $f^{2 n+1}$ and $g^{2 n+1}=\left(g_{k \wedge(2 n+1)}\right)_{k \geq 0}$, satisfies all the assumptions of Section 1. Thus, in order to establish sharpness in Case 2, it is enough to show that $\lim _{n \rightarrow \infty} A_{n}=\infty$. Put $b=(\alpha+1) \eta$. By Taylor's formula, as $n \rightarrow \infty$,

$$
\begin{aligned}
\frac{a_{n+1}}{a_{n}} & =\left(1+\frac{b}{2+n b}\right)^{p-1}\left(1-\frac{r \eta}{2+n b}\right) \\
& =\left(1+\frac{(p-1) b}{2+b n}+O\left(\left(\frac{b}{2+n b}\right)^{2}\right)\right)\left(1-\frac{r \eta}{2+n b}\right) \\
& =\left(1+\frac{p-1}{n}+O\left(\frac{1}{n^{2}}\right)\right)\left(1-\frac{p}{n}+O\left(\frac{1}{n^{2}}\right)\right) \\
& =1-\frac{1}{n}+O\left(\frac{1}{n^{2}}\right) .
\end{aligned}
$$

Now recall the Gauss test for the convergence of a series of positive numbers:

$$
\text { if } a_{n}>0 \text { and } \frac{a_{n+1}}{a_{n}}=1-\frac{\lambda}{n}+O\left(\frac{1}{n^{2}}\right) \text {, then } \sum a_{n}<\infty \text { if and only if } \lambda>1 \text {. }
$$

In our case, $\lambda=1$ so $\sum a_{n}=\lim A_{n}=\infty$.

This completes the proof that $r-1$ is the best constant in the inequality (1.3).

Remarks. 1. The inequality (1.3) holds even for $\alpha>1$. The proof in Section 3 works provided that $\alpha \leq\left[1+\sqrt{1+(p-1)^{2}}\right] /(p-1)$. For $\alpha>1$ sharpness of $r$ could not be established.

2. If $0<\|f\|_{p}<\infty$, then the inequality (1.3) is strict unless $\alpha=0$ and $p=2$ : this follows as in [3] because $r>2$ unless $\alpha=0$ and $p=2$.

3. The inequality (1.3), or, more precisely, the function $U$ in (2.2), leads to analogous inequalities for Itô processes and smooth functions on Euclidean domains. These follow as in [3]. For example, the condition $|\Delta v| \leq|\Delta u|$ in [3] is replaced 
by $|\Delta v| \leq \alpha|\Delta u|$. The inequality $\|v\|_{p} \leq\left(p^{* *}-1\right)\|u\|_{p}$ in [3] becomes $\|v\|_{p} \leq$ $(r-1)\|u\|_{p}$.

\section{ACKNOWLEDGEMENT}

The author wishes to thank Professor Donald L. Burkholder for his help and encouragement throughout this work.

\section{REFERENCES}

1. D. L. Burkholder, Boundary value problems and sharp inequalities for martingale transforms, Ann. Probab. 12 (1984), 647-702. MR 86b:60080

2. _ـ Sharp inequalities for martingales and stochastic integrals. Colloque Paul Lévy sur les processus stochastiques, Astérisque 157-158 (1988), 75-94. MR 90b:60051

3. $ـ$ Strong differential subordination and stochastic integration, Ann. Probab. 22 (1994), 995-1025. MR 95h:60085

Department of Mathematics, University of Illinois, Urbana, Illinois 61801

E-mail address: choi@math.uiuc.edu

Current address: Global Analysis Research Center, Department of Mathematics, College of Natural Sciences, Seoul National University, Seoul 151-742, South Korea

E-mail address: cchoi@math.snu.ac.kr 